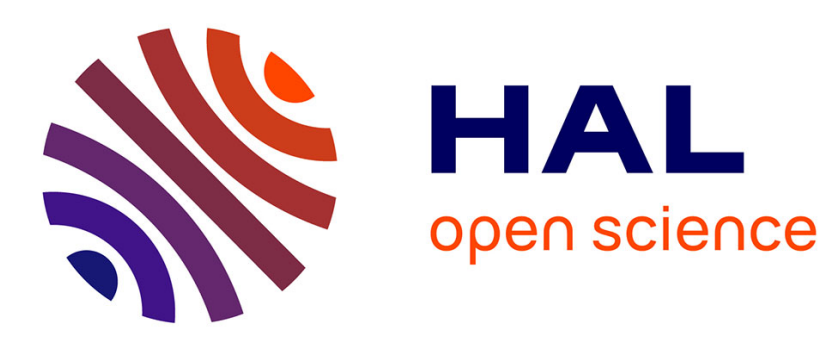

\title{
Linear autocorrelation of partially coherent extreme-ultraviolet lasers: a quantitative analysis
}

Andréa Le Marec, Olivier Larroche, Annie Klisnick

\section{To cite this version:}

Andréa Le Marec, Olivier Larroche, Annie Klisnick. Linear autocorrelation of partially coherent extreme-ultraviolet lasers: a quantitative analysis. Optics Letters, 2017, 42 (23), pp.4958-4961. 10.1364/OL.42.004958 . hal-02397959

\section{HAL Id: hal-02397959 \\ https://hal.science/hal-02397959}

Submitted on 3 Jan 2021

HAL is a multi-disciplinary open access archive for the deposit and dissemination of scientific research documents, whether they are published or not. The documents may come from teaching and research institutions in France or abroad, or from public or private research centers.
L'archive ouverte pluridisciplinaire HAL, est destinée au dépôt et à la diffusion de documents scientifiques de niveau recherche, publiés ou non, émanant des établissements d'enseignement et de recherche français ou étrangers, des laboratoires publics ou privés. 


\title{
Linear autocorrelation of partially coherent XUV laser pulses: a quantitative analysis
}

\author{
Andréa Le MArec ${ }^{1}$, Olivier LARroche ${ }^{2}$, ANd ANNIE KLisnick ${ }^{1}$ \\ ${ }^{1}$ ISMO, Université Paris-Sud, CNRS, Orsay, France \\ ${ }^{2}$ CEA DIF, 91297 Arpajon CEDEX, France \\ *Corresponding author: annie.klisnick@u-psud.fr
}

Compiled September 5, 2017

A quantitative interpretation method is described for experiments involving the linear autocorrelation of partially coherent extreme-ultraviolet (XUV) pulses, generated by either $x$-ray free-electron lasers or plasmabased XUV lasers. A recently published modeling method for partially coherent pulses is numerically implemented in that specific case. Analytical expressions for the statistical root-mean-square average of the fringe visibility curves are derived. The method yields unambiguous informations on both the coherence time and the pulse duration, and should provide a valuable data interpretation tool. (c) 2017 Optical Society of America

OCIS codes: (030.1640) Coherence; (140.2600) Free-electron lasers (FELs); (140.7240) UV, EUV, and X-ray lasers; (340.7450) Xray interferometry.

http://dx.doi.org/10.1364/ol.XX.XXXXXX

The measurement of short partially coherent pulses generated by various kinds of lasers is a long-standing problem [1]. Intensity autocorrelation is a widely used method to measure the duration of ultrashort pulses. It is based on the measurement of nonlinear processes occurring either in crystals, atoms or molecules, where two replicas of the pulse interact with varying delay. As was already discussed in the 70 's $[2,3]$ the partial coherence is responsible for a spiky feature in the autocorrelation trace, appearing at vanishing delay atop the broader, pulselength-related background. The spike, whose width is related to the spectral bandwidth, can be mistaken for the whole pulse, which has led it to be dubbed a "coherent artifact" [4]. In the 80's it was realized that this behavior could be exploited to enhance the temporal resolution in pump-probe measurements (e.g. $[5,6])$ using infrared/visible incoherent light generated from, e.g., incompletely mode-locked lasers. This idea was recently extended to the extreme ultraviolet (XUV) spectral region $[7,8]$ using free-electron lasers (FELs). Due to the amplification of spontaneous emission process FELs intrinsically deliver partially coherent, statistically fluctuating pulses leading to "coherent artifact" features in intensity autocorrelation traces
[9-12] similar to those observed in infrared/visible lasers. Conversely, linear (or field) autocorrelation of a pulse is used to measure its longitudinal coherence, from which the power spectrum and spectral bandwidth of the source can be retrieved. In measurements performed both with XUV FELs [13, 14], and with plasma-based XUV lasers [15, 16] longer features on the order of the pulse duration were observed at delays larger than the coherence time. In analogy with the intensity autocorrelation behavior we propose to call these features a "pulse duration artifact", since it may lead to an erroneously retrieved bandwidth, as was first discussed in Ref. [17]. In this letter, we extend this previous work and describe a detailed quantitative interpretation method for linear autocorrelation measurements of partially coherent pulses, which leads to unambiguous, quantitative information on both the coherence time and the pulse duration.

We start with briefly reviewing the specific experimental data under study. Those include electric field autocorrelation measurements of the coherence time, based on interference fringe visibility, in both XUV FELs and plasma-based XUV lasers. We then investigate the observed behaviour with two complementary methods. First the "Partial Coherence Method" (PCM), a recently introduced [18] heuristic numerical modeling of partially coherent pulses, is reviewed and applied to the present case of electric field autocorrelation. Secondly, we derive an analytical expression of the root-mean-square (rms) average of the modulus of the PCM field autocorrelation function, which gives insight into the experimental visibility measurements. We demonstrate how a modified treatment of the available experimental data could provide a direct comparison with the expression found. Finally our conclusion summarizes the results and touches on future work.

The method of measuring the coherence time by letting a pulse interfere with a time-delayed copy of itself has been implemented in several experiments, both in XUV FEL's and plasma-based XUV lasers. These sources are generated by markedly different techniques : micro-bunching of relativistic electrons in the first case [19]; population inversion in multicharged ions in the second case [20]. They have however in common that the laser emission originates from the amplification of incoherent (noise) emission. This leads to partially coherent output pulses with typical spiky spectral and temporal profiles that fluctuate from shot to 
shot, as will be discussed in more detail below. In this study we specifically consider experiments performed at the FLASH XUV-FEL facility in Hamburg, operating in the selfamplified spontaneous emission (SASE) mode [13, 14], and measurements from two different XUV lasers at Colorado State University, namely a solid-target laser-generated plasma setup [15], operating either in the amplified spontaneous emission (ASE) mode or the high-harmonics (HH) injection-seeded mode, and a capillary discharge plasma ASE setup [16].

The primary data in those experiments are interferograms displaying fringes characterized by a minimum intensity $I_{\min }$ and a maximum intensity $I_{\max }$, which are then translated into values of the visibility $V(\tau)=\left(I_{\max }-I_{\min }\right) /\left(I_{\max }+I_{\min }\right)$ where $\tau$ is the time delay between the two pulse copies. Interferograms are recorded and the visibility is estimated for each individual pulse in a multiple-pulse output, where the individual pulse properties change from pulse to pulse. The visibility values obtained are then averaged over several pulses for each value of the time delay, yielding an average visibility curve $\langle V(\tau)\rangle$. This quantity is proportional to $\langle|\gamma(\tau)|\rangle$, the average modulus of the complex degree of coherence of the source.

Depending on the laser pulse properties, in particular its duration $\Delta t$ and coherence time $\tau_{c}$, the typical shape of the recorded $\langle V(\tau)\rangle$ is a more or less complex peak, which is then tentatively fitted by a sum of one or more gaussian functions. Qualitatively, a single component is found when $\Delta t \gg \tau_{c}$ [16], and more complex shapes including a central peak and a lower and wider "pedestal" are recorded when $\Delta t$ is not much larger than $\tau_{c}$ [15]. As discussed in [17] the central peak and the pedestal can be quantitatively interpreted in terms of these two quantities. In FEL data, the pedestal itself may display some additional structure $[13,14]$. The purpose of the present study is to get a more rigorous and comprehensive interpretation of those results, based on both a numerical implementation and an analytical analysis of the PCM.

Heuristic modeling strategies for partially coherent light have been used in the past, in the case of, e.g., statistical analysis of short pulses from mode-locked lasers [2], or even steady thermal light ([21], § 6.1). Among them, the recently introduced [18] PCM starts from the supposedly known spectral power density of the pulse $\left|\tilde{E}_{0}(\omega)\right|^{2}$ where $\tilde{E}_{0}$ is the field spectral amplitude as a function of angular frequency $\omega$. The width of the spectrum is assumed to be much smaller than its central frequency: $\Delta \omega \ll \omega_{0}$, and since only $\left|\tilde{E}_{0}\right|^{2}$ is known, we will first start from a flat-phase spectral amplitude $\tilde{E}_{0}(\omega)>0$. The associated field in the time domain $E_{0}(t)$ is given by its socalled positive-frequency "analytic signal" ([21], § 3.8): $E_{0}(t)=$ $\operatorname{Re}\left(\mathbf{E}_{0}(t)\right)$ where

$$
\mathbf{E}_{0}(t)=\frac{1}{2 \pi} \int_{-\infty}^{\infty}(1+\operatorname{sign}(\omega)) \tilde{E}_{0}(\omega) \mathrm{e}^{-i \omega t} d \omega
$$

The coherence time $\tau_{c}$ of that Fourier-limited pulse is the width of its analytic signal autocorrelation function ([21], § 5.1)

$$
\Gamma_{0}(\tau)=\int_{-\infty}^{\infty} \mathbf{E}_{0}^{*}(t) \mathbf{E}_{0}(t+\tau) d t
$$

and depends only on the spectral width: $\tau_{c} \propto 1 / \Delta \omega$. A partially coherent signal is obtained from that pulse by applying a random phase factor $\mathrm{e}^{i \varphi_{k}\left(\omega-\omega_{0}\right)}$ to its spectral amplitude. The subscript $k$ identifies the given realization of the random phase function $\varphi_{k}(\xi)$. This operation, since it changes only the spectral phase, preserves the power spectrum, and thus the coherence time, while stretching the pulse in time. Finally the experimentally known pulse duration is recovered by truncating the field by means of a gating function $F_{0}(t)$ of width $\Delta t$. The analytic signal for the resulting partially coherent pulse field then reads

$$
\mathbf{E}_{k}(t)=\hat{\mathbf{E}}_{k}(t) F_{0}(t)
$$

where

$\hat{\mathbf{E}}_{k}(t)=\frac{1}{2 \pi} \mathrm{e}^{-i \omega_{0} t} \int_{-\infty}^{\infty}\left(1+\operatorname{sign}\left(\omega_{0}+\xi\right)\right) \tilde{E}_{0}\left(\omega_{0}+\xi\right) \mathrm{e}^{i \varphi_{k}(\xi)} \mathrm{e}^{-i \xi t} d \xi$

From the analytical autocorrelation function

$$
\Gamma_{k}(\tau)=\int_{-\infty}^{\infty} \mathbf{E}_{k}^{*}(t) \mathbf{E}_{k}(t+\tau) d t
$$

one can then calculate the modulus of the complex degree of coherence $\left|\gamma_{k}(\tau)\right|=\left|\Gamma_{k}(\tau) / \Gamma_{k}(0)\right|$ or the fringe visibility for that pulse. For a given value of the ratio $m=\Delta t / \tau_{c}$, we can plot the average visibility $\langle V(\tau)\rangle=\left\langle\left|\gamma_{k}(\tau)\right|\right\rangle$ as a function of time delay $\tau$, where the angular brackets denote statistical averaging over a large number of realizations $k$ of the random phase function $\varphi_{k}(\xi)$. This is the numerical counterpart of averaging over experimentally measured single-shot visibility values.

We performed a numerical survey following the procedure described above, for several values of $m$ between 3 and 500 , and averaging for each value of $m$ over 1000 random realizations of the phase function $\varphi_{k}(\xi)$ for each discrete frequency $\xi$, using a standard numerical random generator with flat probability density. Gaussian functions are used for the spectrum $\tilde{E}_{0}(\omega)$ and the gating function $F_{0}(t)$. The evolution of the averaged modulus $\left\langle\left|\gamma_{k}(\tau)\right|\right\rangle$ with $m$ is displayed in Fig. 1. For each value of $m$ the calculated curve exhibits a central peak of width comparable to the coherence time $\tau_{c}$, sitting on a lower and wider pedestal. When the ratio $m$ is varied, the relative width $w$ and height $h$ of the pedestal are found to scale as

$$
\begin{aligned}
& w \propto m \tau_{c} \propto \Delta t \\
& h \propto 1 / \sqrt{m} \propto \sqrt{\tau_{c} / \Delta t}
\end{aligned}
$$

This scaling thus clearly relates the two components of the visibility curve to, respectively, the coherence time and the pulse duration, and suggests a method for measuring both at once in a post-processing analysis of a set of experimental single-shot visibility data with varying delay $\tau$ and/or pulse duration $\Delta t$. Preliminary results of such an analysis were presented in Ref. [17]. We note that the fact that the ratio $m$ of pulse duration to coherence time is encoded in both the width and height of the pedestal would lead to a more robust fitting procedure in that analysis, compared to second-order (intensity) autocorrelation traces where the pulse duration is encoded in the width only.

In order to get a more rigorous and comprehensive interpretation of the results of the above numerical study, we present a quantitative analytical analysis of the PCM method applied to field autocorrelation. Definite results are obtained for a quantity closely related (although not identical) to the average fringe visibility, namely the root-mean-square (rms) average of the modulus of the PCM field autocorrelation function $\left\langle\left|\Gamma_{k}(\tau)\right|^{2}\right\rangle^{1 / 2}$. Such a quantity is proportional to the rms average fringe intensity contrast $\left\langle\left(I_{\max }-I_{\min }\right)^{2}\right\rangle^{1 / 2}$ instead of the average visibility $\left\langle\left(I_{\max }-I_{\min }\right) /\left(I_{\max }+I_{\min }\right)\right\rangle$. Our analytical calculations explain the pedestal behaviour while 


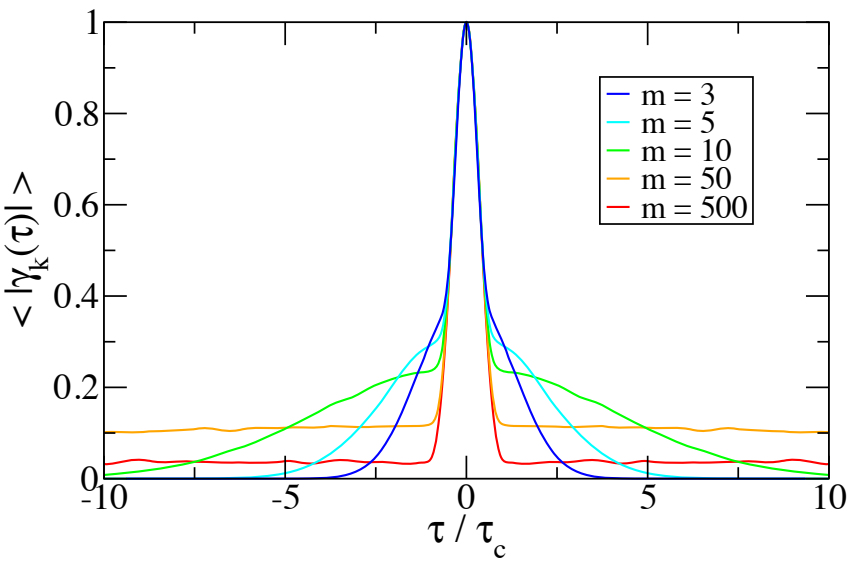

Fig. 1. Modulus of the complex degree of coherence $\left\langle\left|\gamma_{k}(\tau)\right|\right\rangle$ averaged over a large number of realizations $k$, as a function of time delay $\tau$, for 5 values of $m=\Delta t / \tau_{c}$.

providing scaling expressions resembling those previously obtained numerically.

We start from the definition of the autocorrelation function in Eqs. (1)-(3), rewriting Eq. (1) as a convolution product in the frequency domain:

$$
\Gamma_{k}(\tau)=\frac{\mathrm{e}^{-i \omega_{0} \tau}}{2 \pi^{3}} \int_{-\infty}^{\infty} \mathrm{e}^{-i \omega \tau} \iint G_{k}(\xi, \omega)^{*} G_{k}\left(\xi^{\prime}, \omega\right) d \xi d \xi^{\prime} d \omega
$$

where

$$
G_{k}(\xi, \omega)=\tilde{E}_{0}\left(\omega_{0}+\xi\right) \tilde{F}_{0}(\omega-\xi) e^{i \varphi_{k}(\xi)}
$$

and $\tilde{F}_{0}(\omega)$ is the Fourier transform of the gating function $F_{0}(t)$. The squared modulus of $\Gamma_{k}(\tau)$ then reads

$\left|\Gamma_{k}(\tau)\right|^{2}=\frac{1}{4 \pi^{6}} \int_{-\infty}^{\infty} \mathrm{e}^{-i \omega \tau} \int_{-\infty}^{\infty} \mathrm{e}^{i \omega^{\prime} \tau} \iiint \int G_{k}\left(\xi_{1}, \omega\right)^{*} G_{k}\left(\xi_{1}^{\prime}, \omega\right)$

We go over to a discretized version of the integrals over $\xi$ :

$$
\int f(\xi) d \xi \Leftrightarrow \delta \omega \sum_{p=-\frac{N}{2}}^{\frac{N}{2}-1} f(p \delta \omega)
$$

where the relevant frequency interval around $\omega=\omega_{0}$ has been discretized into $N$ bins of width $\delta \omega$ around $\xi=0$. This is actually what is done in the numerical implementation of the PCM procedure, where the frequency increment $\delta \omega$ is a measure of the amount of incoherence imparted to the pulse, and not just an irrelevant numerical parameter. The basic idea in the PCM is to choose a very small (but not vanishing) frequency increment $\delta \omega \ll 1 / \tau_{c}$, which will greatly increase the duration of the pulse, and then cut out from it a part of known duration $\Delta t$. The number of discretization bins $N$ must then be large enough to represent the whole pulse spectrum: $N \delta \omega \gg 1 / \tau_{c}$.

We see that the quadruple integral over $\xi_{1}, \xi_{1}^{\prime}, \xi_{2}$ and $\xi_{2}^{\prime}$ in Eq. (4) can be split up into three sets of terms in the discretized version:

- those for which $p_{1}=p_{1}^{\prime}$ and $p_{2}=p_{2}^{\prime}$, and thus $\varphi_{k}\left(\xi_{1}\right)=$ $\varphi_{k}\left(\xi_{1}^{\prime}\right)$ and $\varphi_{k}\left(\xi_{2}\right)=\varphi_{k}\left(\xi_{2}^{\prime}\right)$ for all $k$;

- those for which $p_{1}=p_{2}$ and $p_{1}^{\prime}=p_{2}^{\prime}$, and thus $\varphi_{k}\left(\xi_{1}\right)=$ $\varphi_{k}\left(\xi_{2}\right)$ and $\varphi_{k}\left(\xi_{1}^{\prime}\right)=\varphi_{k}\left(\xi_{2}^{\prime}\right)$ for all $k$;
- and those for which the phase difference $\varphi_{k}\left(\xi_{1}^{\prime}\right)-$ $\varphi_{k}\left(\xi_{1}\right)+\varphi_{k}\left(\xi_{2}\right)-\varphi_{k}\left(\xi_{2}^{\prime}\right)$ can take any value for different realizations $k$

Obviously each one of the first two sets of terms will add up to the same definite value irrespective of the realization $k$, whereas the third one will add up to a realization-dependent, randomly phased value. The statistical average of $\left|\Gamma_{k}(\tau)\right|^{2}$ over $k$ will thus retain the first two sets of terms only. Re-writing those sums in the form of integrals, this reads

$$
\left\langle\left|\Gamma_{k}(\tau)\right|^{2}\right\rangle=\frac{\delta \omega^{2}}{4 \pi^{2}}\left[\left|\Gamma_{0}(\tau)\right|^{2} \Phi_{0}(\tau)^{2}+\frac{1}{\pi^{4}} \int_{-\infty}^{\infty} \mathrm{e}^{-i \omega \tau} \int_{-\infty}^{\infty} \mathrm{e}^{i \omega^{\prime} \tau}\left(\int \left[\tilde { E } _ { 0 } \left(\omega_{0}+\right.\right.\right.\right.
$$

where

$$
\Phi_{0}(\tau)=\int_{-\infty}^{\infty} F_{0}(t) F_{0}(t+\tau) d t
$$

is the autocorrelation function of the gating function.

Choosing a gaussian spectrum

$$
\tilde{E}_{0}(\omega) \propto \exp \left(-\frac{\tau_{c}^{2}\left(\omega-\omega_{0}\right)^{2}}{8}\right)
$$

and a gaussian gating function

$$
F_{0}(t) \propto \exp \left(-\frac{2 t^{2}}{m^{2} \tau_{c}^{2}}\right)
$$

and inserting those into Eq. (5), we obtain:

$$
\left\langle\left|\Gamma_{k}(\tau)\right|^{2}\right\rangle \propto \exp \left(-\frac{2 \tau^{2}}{m^{2} \tau_{c}^{2}}\right)\left(\exp \left(-\frac{2 \tau^{2}}{\tau_{c}^{2}}\right)+\frac{1}{\sqrt{1+m^{2}}}\right)
$$

It can be seen that $\left\langle\left|\Gamma_{k}(\tau)\right|^{2}\right\rangle$ includes the sum of two gaussian components. The first one corresponds to the central peak of width $\propto \tau_{c}$, while the second relates to the pedestal with a larger width $\propto \Delta t=m \tau_{c}$. After normalization the rms expression

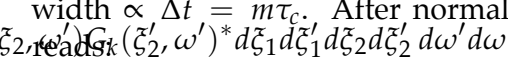

$$
\left(\left\langle\left|\Gamma_{k}(\tau)\right|^{2}\right\rangle /\left\langle\left|\Gamma_{k}(0)\right|^{2}\right\rangle\right)^{1 / 2}=\exp \left(-\frac{\tau^{2}}{m^{2} \tau_{c}^{2}}\right)\left(\frac{1+\sqrt{1+m^{2}} \exp \left(-\frac{2 \tau^{2}}{\tau_{c}^{2}}\right)}{1+\sqrt{1+m^{2}}}\right.
$$

When plotting the evolution of $\left(\left\langle\left|\Gamma_{k}(\tau)\right|^{2}\right\rangle /\left\langle\left|\Gamma_{k}(0)\right|^{2}\right\rangle\right)^{1 / 2}$ with $m$ we find pedestal scalings resembling those previously obtained from the numerical simulations for the averaged modulus of the complex degree of coherence (see Fig. 1) : namely $w=m \tau_{c}$ and $h=\left(1+\sqrt{1+m^{2}}\right)^{-1 / 2}$, instead of $m^{-1 / 2}$ found in the numerical study.

Let us point out that the partially coherent pulse generated in the above-described process $E_{k}(t)=\operatorname{Re}\left(\hat{\mathbf{E}}_{k}(t) F_{0}(t)\right)$ as given by Eqs. (1)-(2) can never strictly recover the Fourierlimited pulse $E_{0}(t)$, whatever value is used for $m$, in contrast to what is stated in Ref. [18]. An improved, iterative PCM procedure allowing to recover the fully coherent initial pulse in the relevant limit is investigated in Ref. [22].

The same expression (7) of the rms field autocorrelation function for gaussian inputs is recovered in a simpler way if partial incoherence is introduced in the time domain instead of the spectral domain, by defining

$$
\mathbf{E}_{k}(t) \propto F_{0}(t) \int_{-\infty}^{\infty} \mathrm{e}^{i \varphi_{k}(u)} \mathbf{E}_{0}(t-u) d u
$$

instead of Eqs. (1)-(2), using the same reasoning to calculate the integrals involved in $\left\langle\left|\Gamma_{k}(\tau)\right|^{2}\right\rangle$ as in the above discussion, 
however now in the time domain with a discretization increment $\delta t$ instead of the frequency domain with a discretization increment $\delta \omega$. Eq. (8) can be interpreted as a superposition of coherent wavepackets with coherence time $\tau_{c}$, each with a given time delay and a random phase shift, the result being then truncated to the prescribed overall pulse duration $\Delta t$.

In addition, defining the partially coherent pulse as in Eq. (8), one can be readily calculate the intensity autocorrelation function

$$
\left\langle\Gamma_{k}^{(4)}(\tau)\right\rangle=\left\langle\mathbf{E}(t) \mathbf{E}^{*}(t) \mathbf{E}(t+\tau) \mathbf{E}^{*}(t+\tau)\right\rangle
$$

using the same arguments for the statistical averages than for $\left\langle\left|\Gamma_{k}(\tau)\right|^{2}\right\rangle$. The obtained expression

$$
\left\langle\Gamma_{k}^{(4)}(\tau)\right\rangle=\delta t^{2} \int_{-\infty}^{\infty} F_{0}(t)^{2} F_{0}(t+\tau)^{2} d t\left(\Gamma_{0}(0)^{2}+\left|\Gamma_{0}(\tau)\right|^{2}\right)
$$

is, apart from notation changes, identical to Eq. (7) of Ref. [2] In the specific case of a gaussian spectrum and pulse shape that expression reads:

$$
\left\langle\Gamma_{k}^{(4)}(\tau)\right\rangle \propto \exp \left(-\frac{2 \tau^{2}}{m^{2} \tau_{c}^{2}}\right) \frac{1}{2}\left(1+\exp \left(-\frac{2 \tau^{2}}{\tau_{c}^{2}}\right)\right)
$$

Such a simple analytical expression could be used to interpret the results of Refs. [7, 11], which supposedly rely on secondorder intensity correlation ( $\Leftrightarrow$ fourth-order field correlation).

In summary, we demonstrated how the pulse duration $\Delta t$ is quantitatively encoded in electric field autocorrelation measurements of the output of SASE X-FEL's and ASE XUV lasers, all of which are stochastic sources with partial coherence: $\Delta t \gtrsim \tau_{c}$. Our results can provide the basis for a simultaneous diagnostic of pulse duration and coherence time ( $\Leftrightarrow$ spectral width), which should be easier to implement than the complex nonlinear processes needed for intensity autocorrelation experiments.

Future work on the theoretical part should investigate the more complex average visibility curves found in $\mathrm{X}$ FEL studies, displaying, e.g., asymmetrical or non-gaussian (multiple-bump) profiles. Higher-order field correlation might be studied, beyond the fourth-order result mentioned at the end of the present work. Spectral shapes more general than simple Gaussians (e.g. Lorentzian or Voigt profiles) could maybe also be included. A direct calculation of the average visibility curve (and not just the rms average as in the present study) would be valuable but seems much more complicated to perform.

Future experimental work will be undertaken at the LASERIX facility in Université Paris-Sud, France, involving linear autocorrelation mesurements in ASE XUV lasers with different pulse durations.

Funding This project has received support from LaserlabEurope (EU-H2020 654148).

Acknowledgment We wish to thank P. Chavel for useful discussions in connection with this work. 


\section{FULL REFERENCES}

1. R. Trebino, Frequency-Resolved Optical Gating: The Measurement of Ultrashort Laser Pulses (Springer Science+Business Media, New York, 2000).

2. H. A. Pike and M. Hercher, "Basis for picosecond structure in modelocked laser pulses," J. Appl. Phys. 41, 4562-4565 (1970).

3. D. B. McDonald, J. L. Rossel, and G. R. Fleming, "Temporal and spectral studies of a synchronously pumped dye laser: Detailed interpretation of autocorrelation measurements," IEEE J. Quantum Electron. QE-17, 1134-1138 (1981).

4. J. Ratner, G. Steinmeyer, T. C. Wong, R. Bartels, and R. Trebino, "Coherent artifact in modern pulse measurements," Opt. Lett. 37, 28742876 (2012)

5. M. Tomita and M. Matsuoka, "Ultrafast pump-probe measurement using intensity correlation of incoherent light," J. Opt. Soc. Am. B 3, 560-563 (1986).

6. J. Y. Huang, Z. P. Chen, and A. Lewis, "Time-delayed secondharmonic generation with nanosecond broadband light pulses: Studies of femtosecond dephasing process of a monolayer of adsorbates," Opt. Commun. 67, 152-158 (1988).

7. K. Meyer, C. Ott, P. Raith, A. Kaldun, Y. Jiang, A. Senftleben, M. Kurka R. Moshammer, J. Ullrich, and T. Pfeifer, "Noisy optical pulses enhance the temporal resolution of pump-probe spectroscopy," Phys. Rev. Lett. 108, 098302 (2012).

8. K. Meyer, N. Müller, Z. Liu, and T. Pfeifer, "Temporal resolution beyond the average pulse duration in shaped noisy-pulse transient absorption spectroscopy," Appl. Opt. 55, 10318-10322 (2016).

9. R. Mitzner, A. A. Sorokin, B. Siemer, S. Roling, M. Rutkowski, H. Zacharias, M. Neeb, T. Noll, F. Siewert, W. Eberhardt, M. Richter, P. Juranic, K. Tiedtke, and J. Feldhaus, "Direct autocorrelation of soft$\mathrm{X}$-ray free-electron-laser pulses by time-resolved two-photon double ionization of He," Phys. Rev. A 80, 025402 (2009).

10. Y. H. Jiang, T. Pfeifer, A. Rudenko, O. Herrwerth, L. Foucar, M. Kurka, K. U. Kühnel, M. Lezius, M. F. Kling, X. Liu, K. Ueda, S. Düsterer, R. Treusch, C. D. Schröter, R. Moshammer, and J. Ullrich, "Temporal coherence effects in multiple ionization of $\mathrm{N}_{2}$ via XUV pump-probe autocorrelation," Phys. Rev. A 82, 041403 (2010).

11. R. Moshammer, T. Pfeifer, A. Rudenko, Y. H. Jiang, L. Foucar M. Kurka, K. U. Kühnel, C. D. Schröter, J. Ullrich, O. Herrwerth, M. F. Kling, X. J. Liu, K. Motomura, H. Fukuzawa, A. Yamada, K. Ueda, K. L. Ishikawa, K. Nagaya, H. Iwayama, A. Sugishima, Y. Mizoguchi, S. Yase, M. Yao, N. Saito, A. Belkacem, M. Nagasono, A. Higashiya, M. Yabashi, T. Ishikawa, H. Ohashi, H. Kimura, and T. Togashi, "Secondorder autocorrelation of XUV FEL pulses via time resolved two-photon single ionization of He," Opt. Express 19, 21698-21706 (2011).

12. A. Senftleben, T. Pfeifer, K. Schnorr, K. Meyer, Y. H. Jiang, A. Rudenko, O. Herrwerth, L. Foucar, M. Kurka, K. U. Kühnel, M. Kübel, M. F. Kling, A. Yamada, K. Motomura, K. Ueda, R. Treusch, C. D. Schröter, R. Moshammer, and J. Ullrich, "Characterization of extreme ultraviolet free-electron laser pulses by autocorrelation," in "Multiphoton Processes and Attosecond Physics,", vol. 125 of Springer Proceedings in Physics (2012), pp. 61-68

13. R. Mitzner, B. Siemer, M. Neeb, T. Noll, F. Siewert, S. Roling, M. Rutkowski, A. A. Sorokin, M. Richter, P. Juranic, K. Tiedtke, J. Feldhaus, W. Eberhardt, and H. Zacharias, "Spatio-temporal coherence of free electron laser pulses in the soft x-ray regime," Opt. Express 16, 19909-19919 (2008)

14. A. Singer, F. Sorgenfrei, A. P. Mancuso, N. Gerasimova, O. M Yefanov, J. Gulden, T. Gorniak, T. Senkbeil, A. Sakdinawat, Y. Liu, D. Attwood, S. Dziarzhytski, D. D. Mai, R. Treusch, E. Weckert, T. Salditt, A. Rosenhahn, W. Wurth, and I. A. Vartanyants, "Spatial and temporal coherence properties of single free-electron laser pulses," Opt. Express 20, 17480-17495 (2012).

15. L. M. Meng, D. Alessi, O. Guilbaud, Y. Wang, M. Berrill, B. Luther, S. R. Domingue, D. H. Martz, D. Joyeux, S. D. Rossi, J. J. Rocca, and A. Klisnick, "Temporal coherence and spectral linewidth of an injectionseeded transient collisional soft x-ray laser," Opt. Express 19, 1208712092 (2011).
16. L. Urbanski, M. C. Marconi, L. M. Meng, M. Berrill, O. Guilbaud, A. Klisnick, and J. J. Rocca, "Spectral linewidth of a Ne-like Ar capillary discharge soft $\mathrm{x}$-ray laser and its dependence on amplification beyond gain-saturation," Phys. Rev. A 85, 033837 (2012).

17. A. L. Marec, O. Guilbaud, O. Larroche, and A. Klisnick, "Evidence of partial temporal coherence effects in the linear autocorrelation of extreme ultraviolet laser pulses," Opt. Lett. 41, 3387-3390 (2016).

18. T. Pfeifer, Y. Jiang, S. Düsterer, R. Moshammer, and J. Ullrich, "Partialcoherence method to model experimental free-electron laser pulse statistics," Opt. Lett. 35, 3441-3443 (2010).

19. J. Feldhaus, J. Arthur, and J. B. Hastings, "X-ray free-electron lasers," J. Phys. B: At. Mol. Opt. Phys. 38, S799-S819 (2005)

20. S. Suckewer and P. Jaeglé, "X-ray laser: past, present, and future," Laser Phys. Lett. 6, 411-436 (2009).

21. J. W. Goodman, Statistical Optics (Wiley, 1985).

22. L. Ahad, I. Vartiainen, T. Setälä, A. T. Friberg, C. David, M. Makita, and J. Turunen, "On spectral and temporal coherence of $\mathrm{x}$-ray free-electron laser beams," Opt. Express 24, 13081-13090 (2016). 